\title{
Polaron-assisted Charge Transport in Li-Ion Battery Anode materials
}

\author{
Matthias Kick ${ }^{\dagger}$ Christoph Scheurer ${ }^{\ddagger}$ and Harald Oberhofer ${ }^{*} \dagger$ \\ $\dagger$ Chair for Theoretical Chemistry and Catalysis Research Center, Technical University of \\ Munich, Lichtenbergstr. 4, 85747 Garching, Germany \\ $\ddagger$ Fritz-Haber-Institut der Max-Planck-Gesellschaft, Faradayweg 4-6, D-14195 Berlin, \\ Germany \\ E-mail: matthias.kick@tum.de
}

\section{Localizing Polarons}

Formally, removing one oxygen atom from the simulation cell will give rise to two excess electrons. These electrons can localize at various different Ti sites, leading to the formation of polaronic $\mathrm{Ti}^{3+}$ centers. ${ }^{1}$ However, different localization patterns usually vary only a little in their total energy, making it challenging to localize the electrons on specific atoms. To this end, we employ the so called matrix control approach. ${ }^{2}$ Here, one starts with a predefined $\mathrm{DFT}+\mathrm{U}$ occupation matrix for the atoms one would like to have the polaron localized on. The occupation matrix elements are chosen in order to resemble the configuration of having a polaron sitting on this atom. For example this can be achieved by changing one of the diagonal elements of this occupation matrix to 1, i.e. representing a fully localized electron. In order to localize the excess electron, the entire occupation matrix is kept fixed during the SCF-cycle. This gives rise to a bias potential which is acting on the electron density in such a way that the electron density adapts to the predefined occupation matrix configuration. 
Note that this is somwhat similar to a constrained DFT treatment. ${ }^{3}$ With the thus biased electron density we then perform a geometry optimization to let the nuclei adapt to this local perturbation, thereby forming a polaron. After this step we used the obtained geometry and wavefunctions information as input for a new calculation without any constraints regarding the occupation matrix.

The above described procedure defines the initial and final states for our nudged elastic band (NEB) calculations. In order to ensure that polarons are always localized at the same atom during the NEB calculations we again make use of the matrix control approach. We start with the obtained self-consistent occupation matrix of our initial state. This occupation matrix serves as an input for each image along the reaction path. However, instead of fixing the occupation matrix for the entire run we only fix the occupation matrix until a certain convergence criterion - slightly higher than the convergence criteria for the scf-cycle itself - is met. This ensures that the electron density is preconverged towards the correct polaronic configuration. After this point we lift the constraint on the occupation matrix and all NEB images are calculated self-consistently. 


\section{Barrier height values}

In Table 1 we list all barrier heights obtained for our systems with oxygen vacancy. Table 2 shows all results for the pristine bulk system with an additional electron yielding a negatively charged cell. In Table 3 we list all barrier heights obtained for the lithiated simulation cells.

Table 1: Obtained barrier heights. $\mathrm{I} \longrightarrow \mathrm{F}$ and $\mathrm{F} \longrightarrow \mathrm{I}$ denote the barrier height going from initial to final state and from final to initial state respectively.

\begin{tabular}{c|c|cc}
\hline \hline bulk & & $\Delta \mathrm{E}_{\mathrm{I} \longrightarrow \mathrm{F}}[\mathrm{eV}]$ & $\Delta \mathrm{E}_{\mathrm{F} \rightarrow \mathrm{I}}[\mathrm{eV}]$ \\
\hline \multirow{4}{*}{$1 \times \mathrm{V}_{\mathrm{O}}$} & $\mathrm{A}-\mathrm{C}$ & 0.368 & 0.357 \\
& $\mathrm{~A}-\mathrm{B}$ & 0.359 & 0.375 \\
& $\mathrm{~A}-\mathrm{B}(\mathrm{q}=+2)$ & 0.415 & 0.414 \\
& pristine & 0.412 & 0.416 \\
& $\mathrm{~A}-\mathrm{B}-\mathrm{C}-\mathrm{E}$ & 0.493 & 0.402 \\
\hline \multirow{3}{*}{$2 \times \mathrm{V}_{\mathrm{O}}$} & $\mathrm{A}-\mathrm{B}-\mathrm{C}-\mathrm{D}$ & 0.481 & 0.346 \\
& $\mathrm{q}=+4$ & 0.552 & 0.368 \\
surface & & & 0.412 \\
\hline \multirow{3}{*}{ LTO $(111)$} & $\mathrm{q}=+2$ & 0.387 & 0.302 \\
& pristine & 0.471 & 0.328 \\
\hline \hline
\end{tabular}

Table 2: Obtained barrier heights for the system where an additional electron is added to the otherwise pristine bulk LTO simulation cell. $\mathrm{I} \longrightarrow \mathrm{F}$ and $\mathrm{F} \longrightarrow \mathrm{I}$ denote the barrier height going from initial to final state and from final to initial state respectively.

\begin{tabular}{c|c|cc}
\hline \hline & & $\Delta \mathrm{E}_{\mathrm{I} \longrightarrow \mathrm{F}}[\mathrm{eV}]$ & $\Delta \mathrm{E}_{\mathrm{F} \rightarrow \mathrm{I}}[\mathrm{eV}]$ \\
\hline \multirow{2}{*}{$\mathrm{q}=-1$} & $\mathrm{~A}$ & 0.369 & 0.372 \\
& $\mathrm{C}$ & 0.392 & 0.402 \\
\hline pristine & & 0.393 & 0.402 \\
\hline \hline
\end{tabular}


Table 3: Obtained barrier heights for the lithiated $2 \times 3 \times 1$ bulk simulation cell. $\mathrm{I} \longrightarrow \mathrm{F}$ and $\mathrm{F} \longrightarrow \mathrm{I}$ denote the barrier height going from initial to final state and from final to initial state respectively.

\begin{tabular}{c|c|cc}
\hline \hline & & $\Delta \mathrm{E}_{\mathrm{I} \rightarrow \mathrm{F}}[\mathrm{eV}]$ & $\Delta \mathrm{E}_{\mathrm{F} \rightarrow \mathrm{I}}[\mathrm{eV}]$ \\
\hline$+\mathrm{Li}$ & $\mathrm{A}$ & 0.373 & 0.354 \\
\hline \multirow{2}{*}{$+2 \mathrm{Li}$} & $\mathrm{A}-\mathrm{B}$ & 0.341 & 0.363 \\
& $\mathrm{q}=+2$ & 0.404 & 0.404 \\
\hline pristine & & 0.393 & 0.402 \\
\hline \hline
\end{tabular}

\section{$\mathrm{U}$ dependence of barrier heights}

In Figure 1 we show how our obtained barrier heights depend on the applied U value. For this analysis we make use of the pristine and A-C barrier profile obtained for the $2 \times 3 \times 1$ bulk system containing a single oxygen vacancy at a $U$ value of $2.65 \mathrm{eV}$. We change the $U$ value from $\mathrm{U}=2.45 \mathrm{eV}$ to $\mathrm{U}=2.85 \mathrm{eV}$ by using the same structures obtained for $2.65 \mathrm{eV}$. At this point one could argue that a $\mathrm{U}$ value of range of $0.4 \mathrm{eV}$ is not significant. However, we would like to point out that this is the consequence of the very localized nature of the Hubbard projector functions used within FHI-aims. As a result, far smaller U values need to be applied as compared to other electronic structure codes. ${ }^{4}$ By varying the $U$ value in total by only $0.4 \mathrm{eV}$ the gap state position for the polaron state — highest lying in energyin A-C changes from $0.88 \mathrm{eV}(\mathrm{U}=2.45 \mathrm{eV})$ to $1.24 \mathrm{eV}(\mathrm{U}=2.85 \mathrm{eV})$ below the conduction band minimum (CBM). At the same time we observe that different $\mathrm{U}$ values have only little influence on our calculated barrier heights. This is not suprisingly at all as the main effect of the Hubbard correction is to shift the gap state up and down in energy. As the same $\mathrm{U}$ value is applied along the entire Li migration path, polaronic states are always subject to the same correction potential as long as the DFT+U occupation matrix does not change significantly. 


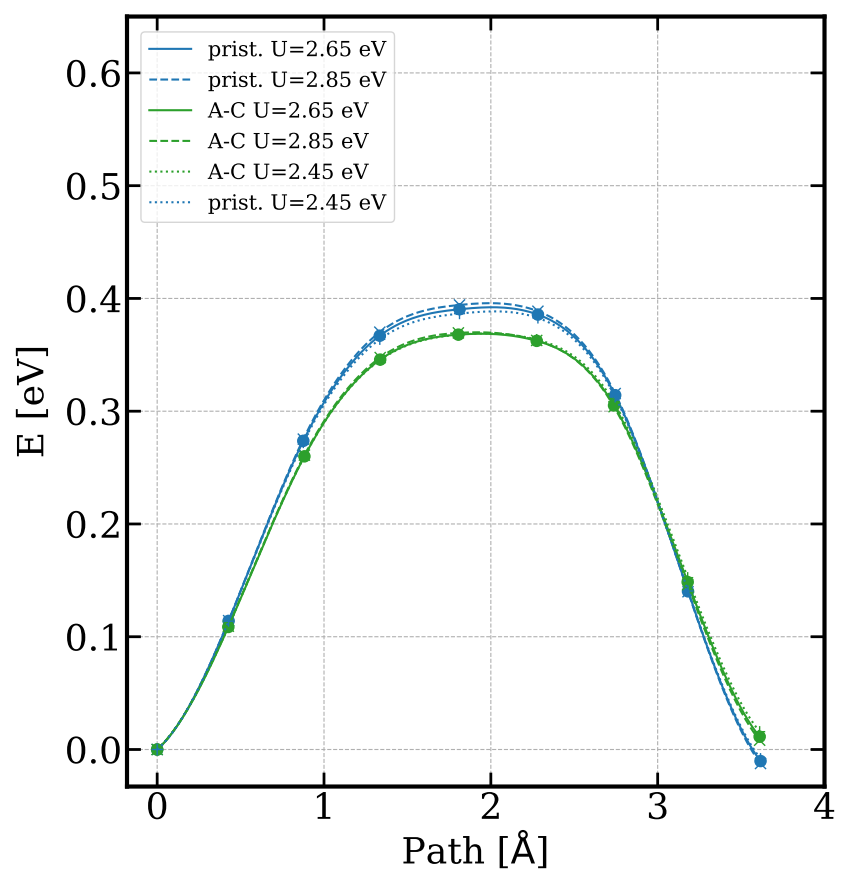

Figure 1: $\mathrm{U}$ dependence of the obtained barrier heights for the $2 \times 3 \times 1$ bulk LTO cell containing a single oxygen vacancy. 


\section{Convergence with system size}

Figure 2 shows the calculated pristine barrier height for a $2 \times 6 \times 1$ bulk LTO cell. We obtain a barrier height of $406 \mathrm{meV}$ if going from initial to final state. This value is in very good agreement with the value for the pristine barrier of the $2 \times 3 \times 1$ bulk LTO cell. See also Table 4 for comparison. The more isolated nature of the Li migration in $2 \times 6 \times 1$-in principle one needs to account for two migration processes in the $2 \times 6 \times 1$ for an exact comparison with the $2 \times 3 \times 1$ cell - explains the little differences between $2 \times 3 \times 1$ and $2 \times 6 \times 1$.

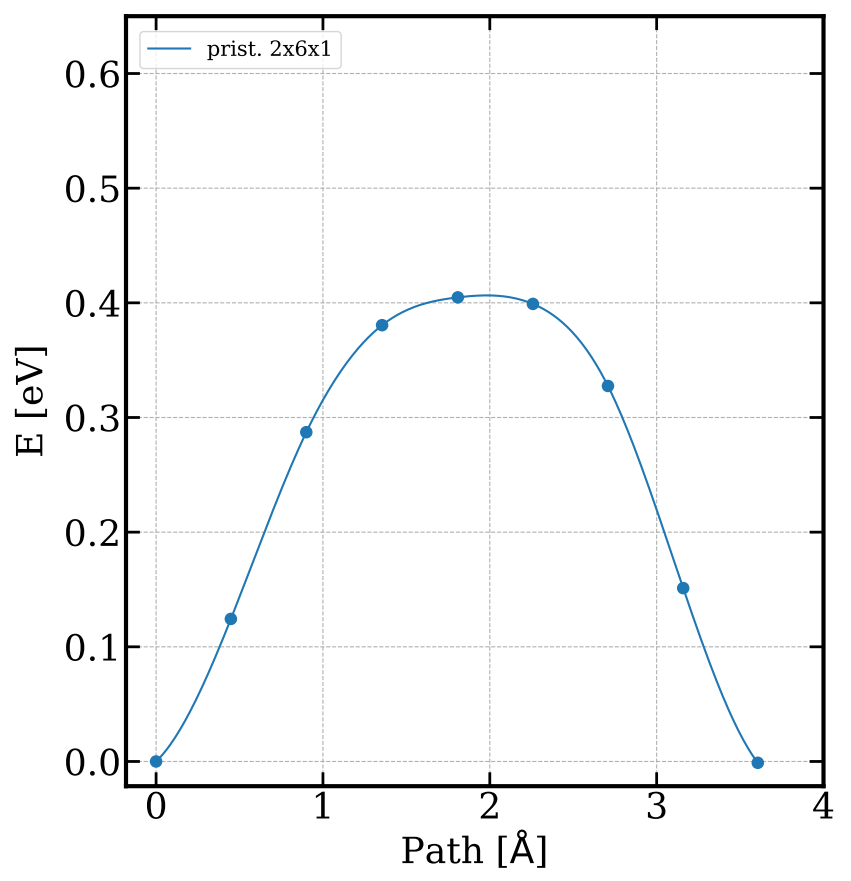

Figure 2: Calculated pristine barrier height for a pristine $2 \times 6 \times 1$ bulk LTO cell.

Table 4: Obtained barrier heights for both the $2 \times 6 \times 1$ pristine bulk LTO cell and for the $2 \times 3 \times 1$ pristine bulk LTO cell. $\mathrm{I} \longrightarrow \mathrm{F}$ and $\mathrm{F} \longrightarrow$ I denote the barrier height going from initial to final state and from final to initial state respectively.

\begin{tabular}{c|c|cc}
\hline \hline bulk & & $\Delta \mathrm{E}_{\mathrm{I} \longrightarrow \mathrm{F}}[\mathrm{eV}]$ & $\Delta \mathrm{E}_{\mathrm{F} \rightarrow \mathrm{I}}[\mathrm{eV}]$ \\
\hline $2 \times 3 \times 1$ & pristine & 0.393 & 0.402 \\
\hline $2 \times 6 \times 1$ & pristine & 0.406 & 0.407 \\
\hline \hline
\end{tabular}




\section{Mobility model}

We assume a simple transition state theory where the hopping is described by an Arrheniuslike equation: ${ }^{5-7}$

$$
k_{\mathrm{hop}}=\frac{k_{\mathrm{B}} T}{h} e^{-E_{\mathrm{A}} / k_{\mathrm{B}} T}
$$

Here, $k_{\mathrm{B}}, T$, and $h$ denote the Boltzmann constant, the temperature, and Planck's constant, respectively. As a first estimate we here also assume entropic effects to stay roughly constant for the different polaron configurations of a given cell to then essentially cancel in the mobility ratio given in equ. 3 below.

These rate constants can then be used in a simple Einstein-Smoluchowsky model of the carrier mobility ${ }^{5}$

$$
\mu=\frac{e a^{2} k_{\mathrm{hop}}}{k_{\mathrm{B}} T}
$$

where $e$ is the elementary charge and $a$ is the distance between the initial and final localization sites.

However, as we are just interested in the ratio between the Li ion mobility in reduced LTO and pristine LTO our equations effectively reduce to,

$$
\frac{\mu^{\text {reduc. }}}{\mu_{\text {prist. }}}=\frac{\frac{e a^{2} k_{\text {hop }}^{\text {reduc. }}}{k_{\mathrm{B}} T}}{\frac{e a^{2} k_{\text {hop }}^{\text {prist. }}}{k_{\mathrm{B}} T}}=\frac{k_{\text {hop }}^{\text {reduc. }}}{k_{\text {hop }}^{\text {prist. }}}
$$

At $\mathrm{T}=300 \mathrm{~K}$ we find an almost fourfold increase $-\mathrm{A}-\mathrm{B}$ system with $\frac{\mu^{\text {reduc. }}}{\mu_{\text {prist. }}}=3.7$ - on introducing a single oxygen vacancy into the pristine $2 \times 3 \times 1$ bulk LTO cell.

\section{References}

(1) Kick, M.; Grosu, C.; Schuderer, M.; Scheurer, C.; Oberhofer, H. Mobile Small Polarons Qualitatively Explain Conductivity in Lithium Titanium Oxide Battery Electrodes. J. Phys. Chem. Lett. 2020, 11, 2535-2540. 
(2) Allen, J. P.; Watson, G. W. Occupation matrix control of d- and f-electron localisations using DFT + U. Phys. Chem. Chem. Phys. 2014, 16, 21016-21031.

(3) Wu, Q.; Van Voorhis, T. Direct optimization method to study constrained systems within density-functional theory. Phys. Rev. A 2005, 72, 024502.

(4) Kick, M.; Reuter, K.; Oberhofer, H. Intricacies of DFT+U, not only in a numeric atom centered orbital framework. J. Chem. Theor. Comput. 2019, 15, 1705-1718.

(5) Oberhofer, H.; Reuter, K.; Blumberger, J. Charge Transport in Molecular Materials: An Assessment of Computational Methods. Chem. Rev. 2017, 117, 10319.

(6) Devreese, J. T. digital Encyclopedia of Applied Physics; American Cancer Society, 2003.

(7) Devreese, J.; on Polarons, A. S. I. F.; in Semiconductors, E. P. I. P. Polarons in Ionic Crystals and Polar Semiconductors; NATO Advanced Study Institutes Series; NorthHolland Publishing Company, 1972. 